\title{
Online Learning of the Dynamical Internal Model of Transfemoral Prosthesis for Enhancing Compliance
}

\author{
Heins, Sophie; Tolu, Silvia; Ronsse, Renaud
}

Published in:

IEEE Robotics and Automation Letters

Link to article, DOI:

10.1109/LRA.2021.3091953

Publication date:

2021

Document Version

Peer reviewed version

Link back to DTU Orbit

Citation (APA):

Heins, S., Tolu, S., \& Ronsse, R. (2021). Online Learning of the Dynamical Internal Model of Transfemoral Prosthesis for Enhancing Compliance. IEEE Robotics and Automation Letters, 6(4), 6156 - 6163.

https://doi.org/10.1109/LRA.2021.3091953

\section{General rights}

Copyright and moral rights for the publications made accessible in the public portal are retained by the authors and/or other copyright owners and it is a condition of accessing publications that users recognise and abide by the legal requirements associated with these rights.

- Users may download and print one copy of any publication from the public portal for the purpose of private study or research.

- You may not further distribute the material or use it for any profit-making activity or commercial gain

- You may freely distribute the URL identifying the publication in the public portal

If you believe that this document breaches copyright please contact us providing details, and we will remove access to the work immediately and investigate your claim. 


\title{
Online Learning of the Dynamical Internal Model of Transfemoral Prosthesis for Enhancing Compliance
}

\author{
Sophie Heins ${ }^{1}$, Silvia Tolu ${ }^{2}$ and Renaud Ronsse ${ }^{1}$
}

\begin{abstract}
Powered prostheses hold promises to restore adaptive and robust locomotion to lower-limb amputees. However, their daily use is still challenged by several shortcomings, on top of which those related to their control methods. This paper reports the development of an adaptive controller for a transfemoral prosthesis that combines a predictive torque component with a feedback error-correction mechanism. The predictive module is based on the Locally Weighted Projection Regression (LWPR) algorithm that achieves nonlinear function approximation of a dynamical model of the prosthesis joints. The performance of the proposed control strategy are assessed with a simulated biped walker with a unilateral transfemoral amputation. Results show that the LWPR-based module provides accurate predictions of the ankle and knee torques, resulting in a precise position tracking. This allows reducing the gains of the feedback error-correction mechanism by one order of magnitude, leading to a feedback contribution to the total joint torque lower than $3 \%$ and $8 \%$ for the ankle and the knee, respectively. Compliance of both prosthesis joints is enhanced accordingly. In addition, the control architecture is robust to speed changes while the joint dynamical internal model is continuously learned. This approach is thus promising for the development of adaptive controllers for lower-limb prostheses.
\end{abstract}

\section{INTRODUCTION}

Lower-limb loss is a strongly incapacitating condition affecting many aspects of life, including mobility, social life, psychological well-being and general discomfort [1]-[3]. To replace their missing limb, amputees can be equipped with a leg prosthesis, which improves their quality of life and helps them recovering some independence. Transfemoral, i.e. above-knee, amputees are often prescribed a semi-passive, microprocessor controlled knee prosthesis (e.g. the Ottobock C-leg [4]) combined with a semi-passive, energy storage and return ankle prosthesis (e.g. the Ossür Proprio foot [5]). However, walking with such a prosthesis requires large energy expenditure and is characterized by slower speed and less stability compared to unimpaired walking. The higher the amputation level, the worse the walking performance [6][8]. Recent evidence showed that lower-limb amputees could benefit from using an active prosthetic device by decreasing

Manuscript received: February 23, 2021; Revised: April 14, 2021; Accepted: June 6, 2021.

This paper was recommended for publication by Editor Pietro Valdastri upon evaluation of the Associate Editor and Reviewers' comments.

This work was supported by the European Community's H2020 Research and Innovation Programme under grant number 731931 (the CYBERLEGsPlusPlus collaborative project).

1 Sophie Heins and Renaud Ronsse are with the Institute of Mechanics, Materials, and Civil Engineering; Institute of Neuroscience; and Louvain Bionics; UCLouvain, Louvain-la-Neuve, Belgium renaud.ronsseduclouvain.be

${ }^{2}$ Silvia Tolu is with the Department of Electrical Engineering; Technical University of Denmark, Copenhagen, Denmark

Digital Object Identifier (DOI): see top of this page. the load of the sound limb during walking [9] or reducing the associated metabolic cost [10]. However, designing such solutions still raises many challenges, related to both their mechanical design (weight or autonomy) and the development of appropriate control strategies for their actuators. The present work focuses on the latter.

Several control frameworks are being investigated in different devices [11], based either on a finite state machine with impedance control [12] or on time- or phase-dependent trajectories to be tracked by the prosthesis joints [13]-[16]. A recent trend consists in adapting these trajectories to the user in realtime, for instance in order to minimize some metabolic metrics [17], [18]. Given the dynamic nature of human locomotion and the periodic and intermittent interactions with the environment, a general design rule is to rely on low-impedance control laws contributing to the user's safety by increasing the controller's robustness and helping to cope with environment uncertainties [11]. Compliance is indeed a fundamental feature of human locomotion [19], [20] and is likely achieved by combining predictive and pure feedback-based control mechanisms [21].

In this work, we report the development of an adaptive controller for a lower-limb prosthesis using an iterative online learning method. This controller combines a predictive contribution with a feedback error-correction mechanism. The predictive module provides an accurate prediction of the control command by using the Locally Weighted Projection Regression (LWPR) algorithm that achieves non-linear function approximation using local linear models [22]-[24]. The complementary feedback pathway can thus have low gains, leading to a resulting low impedance at both joints. In sum, this controller achieves precise tracking of a reference trajectory, while displaying low joint impedance in case of perturbation. Due to the fact that this approach has never been used for the control of actual lower-limb prostheses, and thus for safety reasons, the proposed control strategy was firstly validated on a simulated biped walker with a transfemoral amputation. The material presented here is an improved and extended version of the preliminary study presented in [25], both regarding the methodology and the quality of the results. The paper is structured as follows. Section II outlines the general control architecture. Section III provides a description of the simulation environment used for assessing the controller's performance and develops the experimental procedure. Simulation results are presented in Section IV and discussed in Section V.

\section{CONTROL ARCHItecture}

The proposed control framework is depicted in Fig. 1 and relies on joint position tracking. For each joint of the prosthesis (i.e., ankle and knee), compliant position tracking is achieved 
by combining feedback and predictive torque commands. For each joint $i \in\{a ; k\}$, the desired torque is expressed as $\tau_{\mathrm{des}}^{i}=\tau_{\mathrm{FB}}^{i}+\tau_{\text {pred }}^{i}$, where the feedback torque component $\tau_{\mathrm{FB}}^{i}$ is computed by a proportional-derivative (PD) controller and the predictive torque component $\tau_{\text {pred }}^{i}$ relies on iterative learning of a dynamical internal model. This control architecture - and in particular its prediction module — is inspired from [26], [27].

\section{A. Reference Trajectories}

For each joint $i \in\{a ; k\}$, the reference trajectory $\theta_{\text {ref }}^{i}$ is obtained from a weighted combination of 3 periodic Gaussian functions, which are expressed as:

$$
\mathcal{G}_{n}(\phi)=\exp \left(\frac{\cos \left(2 \pi\left(\phi-\mu_{n}\right)\right)-1}{\sigma_{n}}\right)
$$

where $\mu_{n}$ is the peak position of the $n$-th Gaussian function and $\sigma_{n}$ its width. Their values were selected based on angular trajectories taken from the literature for slow, normal and fast walking, corresponding respectively to a cadence of $0.71,0.83$ and 1 stride/sec [28]. More precisely, the chosen values of $\mu_{n}$ match a joint flexion or extension peak, and they were respectively set to $(0.06 ; 0.44 ; 0.66)$ and $(0.14 ; 0.72 ; 0.98)$ for the ankle and the knee joints; and the values of $\sigma_{n}$ were respectively set to $(0.02 ; 0.62 ; 0.20)$ and $(0.63 ; 0.50 ; 0.12)$, which are associated to the lowest reconstruction error between the obtained reference and the original trajectories. These Gaussian functions are continuous functions of the gait phase $\phi \in[0,1]$ and their weights are modulated as a function of the gait cadence $\omega$. These weights were first computed for slow, normal and fast walking based on the trajectories of [28], and then interpolated by second-order polynomials to make them continuous functions of the gait cadence and thus generating smooth transitions across all walking speeds. The gait phase $\phi$ and cadence $\omega$ are estimated in real-time from the hip angle of the amputated leg $\theta_{h}$ by means of an adaptive oscillator [29]. Approximating the reference trajectories by linear combinations of periodic Gaussian functions aimed at smoothing these trajectories and making them continuously differentiable [30].

\section{B. Predictive contribution: Dynamical Internal Model}

The predictive torque component is computed by an adaptive dynamical internal model of the joint (orange block in Fig. 1). This dynamical model is incrementally learned by LWPR, an algorithm that has been successfully used in different simulation studies, e.g. [26], [27]. In our approach, each prosthesis joint $i \in\{a ; k\}$ has its own LWPR module, computing $\tau_{\text {pred }}^{i}$ from the set of sensory inputs $\mathbf{x}$. These sensory inputs are the reference angular position and velocity of the specific joint $\left(\theta_{\text {ref }}^{i}\right.$ and $\left.\dot{\theta}_{\text {ref }}^{i}\right)$, and the actual angular positions and velocities of the ankle, knee and hip joints of the amputated leg $\left(\theta_{a}, \theta_{k}\right.$, $\theta_{h}$ and $\left.\dot{\theta}_{a}, \dot{\theta}_{k}, \dot{\theta}_{h}\right)$. Importantly, we included neither angular accelerations nor contact forces in the input vector $\mathbf{x}$, although the exact dynamical model of the prosthesis also depends on these variables. Indeed, we decided to restrict the input vector

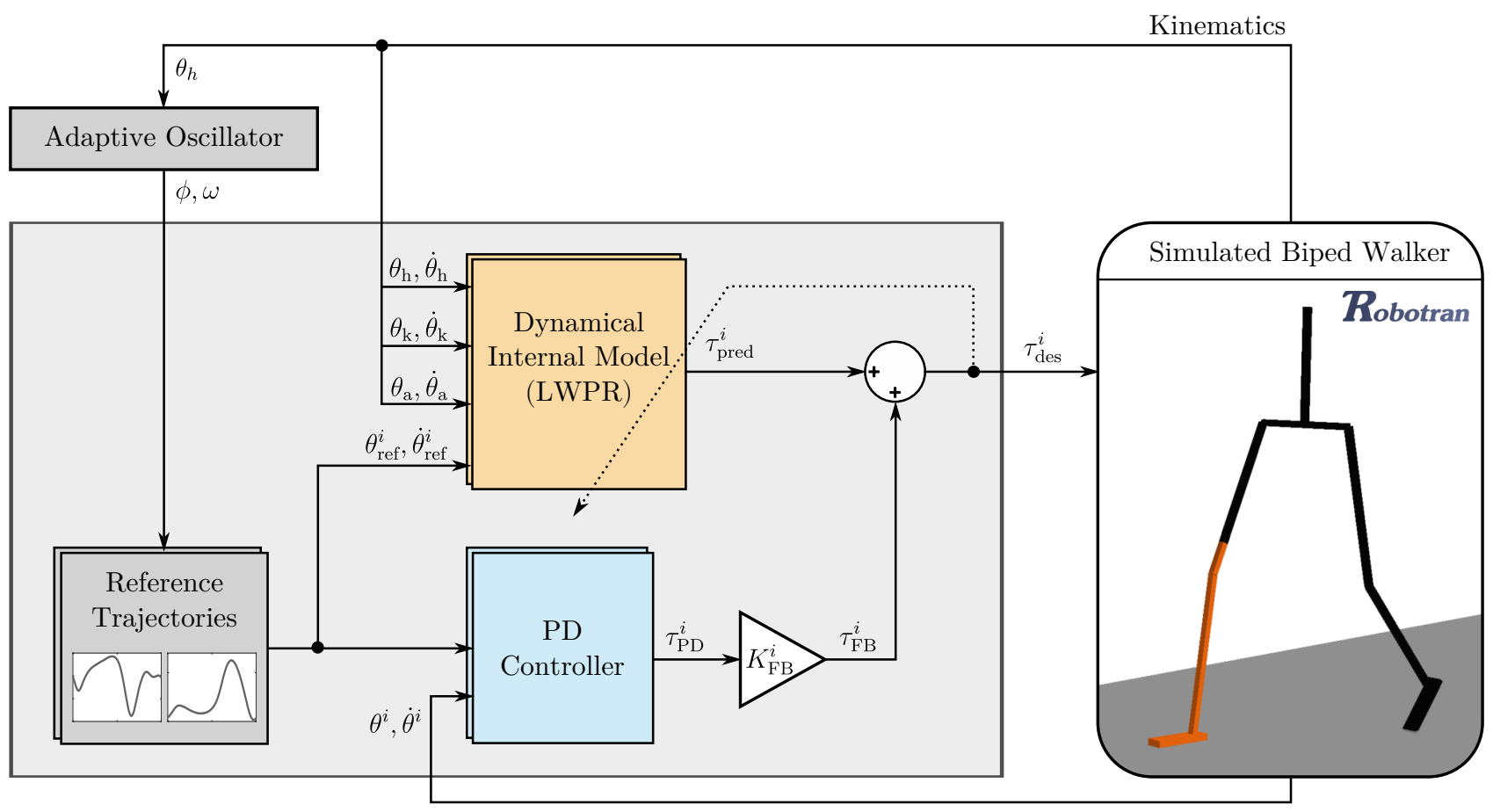

Fig. 1. Global control architecture of a simulated biped walker with transfemoral amputation. The controller combines predictive (orange) and pure feedback (blue) torque components for the ankle and knee joints. The superscript $i \in\{a ; k\}$ refers to the controlled joint. The dynamical internal model of the ankle (resp. knee) receives the reference kinematics of the ankle (resp. knee); while both receive the actual hip, knee, and ankle kinematics. The predictive component $\tau_{\text {pred }}^{i}$ is computed by these dynamical internal models, which are incrementally learned by the LWPR algorithm. The pure feedback component $\tau_{\mathrm{FB}}^{i}$ is computed by a PD controller. 
to signals that could be readily measured on a real device, i.e. having joint encoders but no contact force sensors. The predictive torque is a weighted mean of $k$ local linear models $\psi_{k}(\mathbf{x})$, and the weights $w_{k}(\mathbf{x})$ are Gaussian functions called receptive fields (RFs):

$$
\begin{aligned}
\tau_{\text {pred }}^{i}(\mathbf{x}) & =\sum_{k} w_{k}(\mathbf{x}) \psi_{k}(\mathbf{x}) \\
w_{k}(\mathbf{x}) & =\exp \left(\left(\mathbf{x}-c_{k}\right)^{T} D_{k}\left(\mathbf{x}-c_{k}\right) / 2\right)
\end{aligned}
$$

where $D_{k}$ is the distance metric and $c_{k}$ is the center of each RF. These parameters define the validity region of the local models. The local models (i.e. their regression directions) are continuously updated using weighted partial least square (PLS) regression, and new local models are created if, for a training sample $\mathbf{x}$, no local model has an activation $w_{k}(\mathbf{x})$ above a configurable threshold. The distance metric $D_{k}$ of each RF is continuously adjusted using stochastic cross validation. More details on the LWPR algorithm and its parameters can be found in [31].

\section{Feedback error contribution: PD controller}

The feedback controller (blue block in Fig. 1) provides error correction following the reference trajectory. It is a PD controller, whose gains $K_{p}$ and $K_{d}$ take independent values for the swing phase and for the stance phase. The global gain $K_{\mathrm{FB}}^{i}$ is used to modulate the overall feedback contribution, and in particular to decrease it once the dynamical model is learned in order to enhance the prosthesis compliance.

\section{EXPERIMENTAL METHODS}

This work assesses the ability of the LWPR-based module to act as a dynamical internal model for the knee and ankle joints of a transfemoral prosthesis, based on the experimental methods described in this section.

\section{A. Simulated Biped Walker}

The biped model depicted in Fig. 1 was developed in the Robotran multibody software [32], [33]. It is composed of 7 rigid bodies (a trunk and 2 segmented legs with foot, shank and thigh), 6 revolute joints that only move in the sagittal plane (ankle, knee and hip of both legs), and 1 prismatic joint that only moves in the vertical direction (the center of mass). The dimensions, mass and inertia of the bodies constituting the biped walker were taken from [34], and its total mass is $80 \mathrm{~kg}$. The ground contact model accounts only for the vertical component of the ground reaction force (GRF), and its computation is based on [34]. The hip, knee and ankle joints of the left leg and the hip joint of the right leg are position-controlled, i.e. they perfectly track reference position profiles that are synchronized to a so-called artificial phase variable $\phi_{\text {ref }}=\bmod \left(t, \frac{1}{\omega_{\text {ref }}}\right) \omega_{\text {ref }}$, where $t$ is the simulation time and $\omega_{\text {ref }}$ is the imposed gait cadence. The right hip joint is synchronised with $\phi_{\text {ref }}$, whereas the left hip, knee and ankle joints are synchronized with $\phi_{\text {ref }}$ shifted by $50 \%$. These joints track Winter reference trajectories for slow, normal or fast walking [28], depending on the experimental tests (see
Section III-B). The control architecture presented in Section II is implemented on the simulated prosthetic joint, i.e., the knee and ankle of the right leg (represented in dark orange in Fig. 1). During the simulations, the desired torque patterns were directly fed to these joints, assuming perfect torque tracking, i.e. modelling these joints as pure sources of torque.

\section{B. Experimental protocol}

The performance of the proposed controller architecture was assessed with the simulation tests described below. Experiments 1 and 2 focus on normal walking data only, while experiment 3 specifically focuses on transitions between the three walking cadences.

Experiment 1: Basic normal walking: This experiment aims at evaluating the capacity of the LWPR-based module to act as a dynamical internal model and predict accurate torque commands for the knee and ankle joints of a transfemoral prosthesis. During the task, the dynamical models were initially learned during 2 gait cycles while the prosthesis joints were controlled in pure feedback mode, i.e. while the desired torque was such that: $\tau_{\text {des }}^{i}=\tau_{\mathrm{PD}}^{i}$. These 2 gait cycles of initial training were performed to avoid including in the total torque nonlinearities that affect the prediction and thus the stability of the system. The predictive torque was then included in the desired torque from the beginning of the third gait cycle and the dynamical models were continuously learned until the end of the simulation. The simulation was performed over 100 gait cycles in normal walking conditions.

Experiment 2: Reduced joint impedance: This experiment assesses the feasibility of increasing the joint compliance after the initial learning phase of the dynamical internal models. In other words, it evaluates the feasibility of lowering the prosthesis joints impedance while maintaining a similar tracking accuracy. It is performed in two steps:

A) Reduced PD gains: we first assess the feasibility of decreasing the feedback contribution of the joint torque commands by reducing the feedback controller gains after the initial learning of the dynamical internal models. During this task, the dynamical internal models of the prosthesis joints were initially learned during 50 gait cycles in normal walking conditions (like in Experiment 1). Then, the PD gains were decreased by one order of magnitude, i.e. the value of $K_{\mathrm{FB}}^{i}$ was set to $10 \%$ for both joints. The LWPR training continued for 50 additional gait cycles, i.e., until the end of the simulation. The contributions of the predictive torque and feedback torque components to the total joint torques were assessed by computing the ratio between the integral of their respective absolute value over the cycle.

B) Torque perturbation: we then evaluate the effect of decreasing the PD gains on the joints impedance, by applying a torque perturbation to the prosthesis joints and measuring the resulting angular displacement. More specifically, we compare three cases: (i) the pure feedback mode (i.e. forcing $\tau_{\text {des }}^{i}=\tau_{\mathrm{PD}}^{i}$ ), (ii) the predictive controller combined with high PD gains (i.e., $K_{\mathrm{FB}}^{i}=100 \%$ ), and (iii) the predictive controller combined with low PD gains (i.e., $K_{\mathrm{FB}}^{i}=10 \%$ ). In each case, the biped walker executes one gait cycle during which 
a torque perturbation lasting $50 \mathrm{~ms}$ is applied to the ankle or knee joint at specific gait phases $\phi_{\text {pert }}$. The dynamical internal models learned during Experiments 1 and 2.A were used by the LWPR module in (ii) and (iii) respectively, but their update was stopped to avoid learning the torque perturbation. We tested ankle perturbations of $2 \mathrm{Nm}$ for $\phi_{\text {pert }}=\{0 \%, 75 \%\}$ (i.e. during the swing phase or the swing-to-stance transition) and $50 \mathrm{Nm}$ for $\phi_{\text {pert }}=\{25 \%, 50 \%\}$ (i.e. during the stance phase), and knee perturbations of $65 \mathrm{Nm}$ for $\phi_{\text {pert }}=$ $\{0 \%, 25 \%, 50 \%, 75 \%\}$.

Experiment 3: Cadence switching: The third experiment focuses on evaluating the robustness of the proposed controller to changing walking cadences. In this experiment, the imposed walking cadence $\omega_{\text {ref }}$ was switched every 25 gait cycles, to slow, normal or fast walking, as defined in [28]. Transitions occurred over the first 5 cycles of each new cadence. During these 5 transition cycles, the reference trajectories of the simulated non-prosthetic joints were linearly blended from one to the other, and the prosthesis reference trajectories adapted via weighting of the Gaussian functions from the estimated cadence $\omega$ (see Section II-A). Like in the other experiments, learning of the dynamical internal models started at the beginning of the simulation, but the prosthesis joints were controlled in pure feedback mode during the first 2 gait cycles. Again, this initial learning was performed to avoid including in the total torque nonlinearities that affect the prediction and thus the stability of the system. The cadence sequence was: Normal - Slow - Normal - Fast - Normal Fast - Normal - Slow - Normal - Fast - Normal - Slow. The global feedback gain $K_{\mathrm{FB}}^{i}$ was set to $100 \%$ throughout the experiment, for both joints.

\section{Parameters tuning}

The controller's parameters related to the feedback and to the predictive contributions were tuned empirically in the simulation environment with normal walking conditions, before conducting the experimental tests.

1) Feedback gains: The PD gains $K_{p}$ and $K_{d}$ of the feedback torque were tuned while the prosthesis joints received $100 \%$ of the feedback torque component as control command, i.e. $\tau_{\text {des }}^{i}=\tau_{\mathrm{PD}}^{i}$. The synthesis objective was to maintain trajectory tracking error below $2 \mathrm{deg}$ for the ankle and 6

TABLE I

CONTROLLER PARAMETERS

\begin{tabular}{|c|c|c|c|}
\cline { 2 - 4 } \multicolumn{2}{c|}{ Feedback controller } & Ankle joint & Knee joint \\
\hline \multirow{2}{*}{$K_{p}[\mathrm{Nm} / \mathrm{rad}]$} & Stance & 5000 & 6000 \\
\cline { 2 - 4 } & Swing & 110 & 1000 \\
\hline \multirow{2}{*}{$K_{d}[\mathrm{Nms} / \mathrm{rad}]$} & Stance & 15 & 200 \\
\cline { 2 - 4 } & Swing & 1.25 & 25 \\
\hline \multicolumn{2}{|c|}{ init_D } & 40 & 40 \\
\hline \multicolumn{2}{|c|}{ w_gen } & 0.1 & 0.1 \\
\hline \multicolumn{2}{|c|}{ init_ $\alpha$} & 5 & 10 \\
\hline add_threshold & 0.5 & 0.5 \\
\hline
\end{tabular}

LWPR-based module deg for the knee, i.e. $10 \%$ of their maximum absolute value. Numerical values of the feedback gain are provided in Table I. To ensure a smooth transition between stance and swing, a linear transition lasting $0.02 \mathrm{~s}$ following toe-off and heel strike was implemented.

2) LWPR configuration: The most relevant parameters of the LWPR algorithm were tuned with the objective to cooptimize learning accuracy (i.e. minimizing RMSE between predicted and desired torque profiles) and speed (i.e. minimizing time before reaching stationary behavior). They are reported in Table I, where init_D denotes the initial value of the distance matrix $D_{k}$ (Eq. (3)), w_gen is a threshold value for the RFs $w_{k}$ that determines when a new local model needs to be created, init_ $\alpha$ is the initial learning rate for the gradient descent algorithm that is used to continuously update the RFs distance metric $D_{k}$, and add_threshold is a threshold value determining when a new regression direction should be added. A more detailed definition of each parameter is provided in [31], [35].

\section{Simulation Results}

\section{A. Experiment 1: Basic normal walking}

Results of the basic normal walking experiment showed that the LWPR-based module is able to quickly learn the dynamical internal models of the knee and ankle joints and to provide precise torque commands, producing stable and accurate walking patterns. This is illustrated in Fig. 2 showing the ankle and knee angle profiles averaged over the last 5 cycles of the simulation and compared to the ones obtained with a pure feedback control strategy in (a-b), and their corresponding predicted torque profiles compared to the desired torques in (c-d).

We can observe that the torque predicted by the LWPRbased module is very similar to the total joint torque for both joints. The root mean squared error normalised to the
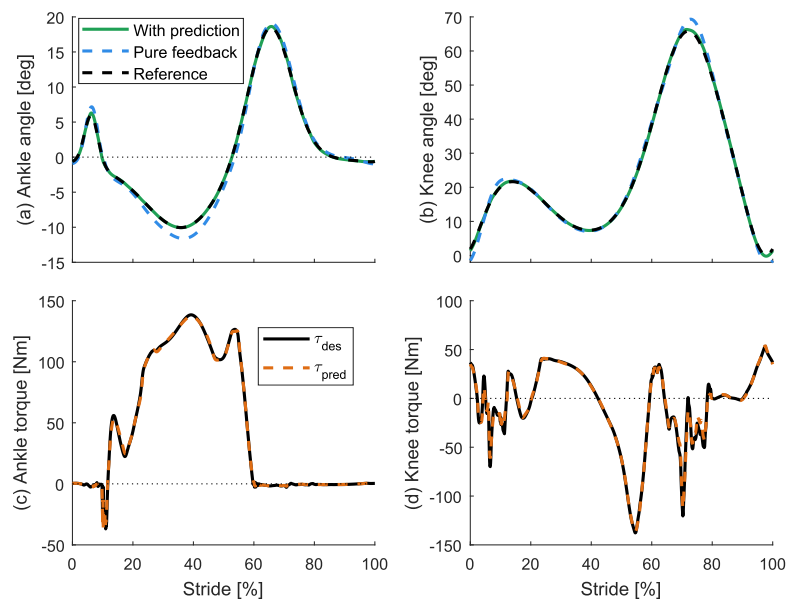

Fig. 2. Experiment 1: Joint position and torque trajectories averaged over the last 5 cycles of simulation: (a)-(b) Ankle and knee angles obtained with both control contributions (feedback and predictive) and compared to their reference positions and to their corresponding profiles obtained in pure feedback mode; (c)-(d) Ankle and knee torque predicted by the LWPR-based module $\left(\tau_{\text {pred }}\right)$ and compared to the total joint torque $\left(\tau_{\text {des }}\right)$. 
maximum amplitude (nRMSE) between the predicted torque and the total torque converges to $0.34 \%$ for the ankle joint and to $1.03 \%$ for the knee joint. This results in accurate tracking of the ankle and knee position reference profiles, and the nRMSE between the joint actual position and its reference trajectory converges to $0.17 \%$ for the ankle joint and to $0.26 \%$ for the knee joint. These values are lower than the ones obtained in pure feedback mode - whose mean value are $2.59 \%$ and $1.28 \%$ for the ankle and the knee joints respectively. During the simulation, 48 and 79 RFs were created by the LWPR algorithm for the ankle model and for the knee model respectively.

\section{B. Experiment 2: Reduced joint impedance gains}

We observed during the second experiment that the proposed controller still achieves a good position tracking accuracy for both joints when reducing the PD gains by one order of magnitude, after sufficient learning. Indeed, the nRMSE of the joint angular positions converges to $0.91 \%$ for the ankle joint and to $0.55 \%$ for the knee joint (see the evolution per cycle in Fig. 3(a)). Although these values are higher than the nRMSE of the joint angular positions observed in Experiment 1 , the tracking performance is still better than the one obtained in pure feedback mode for both joints. In addition, reducing the PD gains allowed to further decrease the contribution of the feedback torque component to the total joint torque. Fig. 3(b)
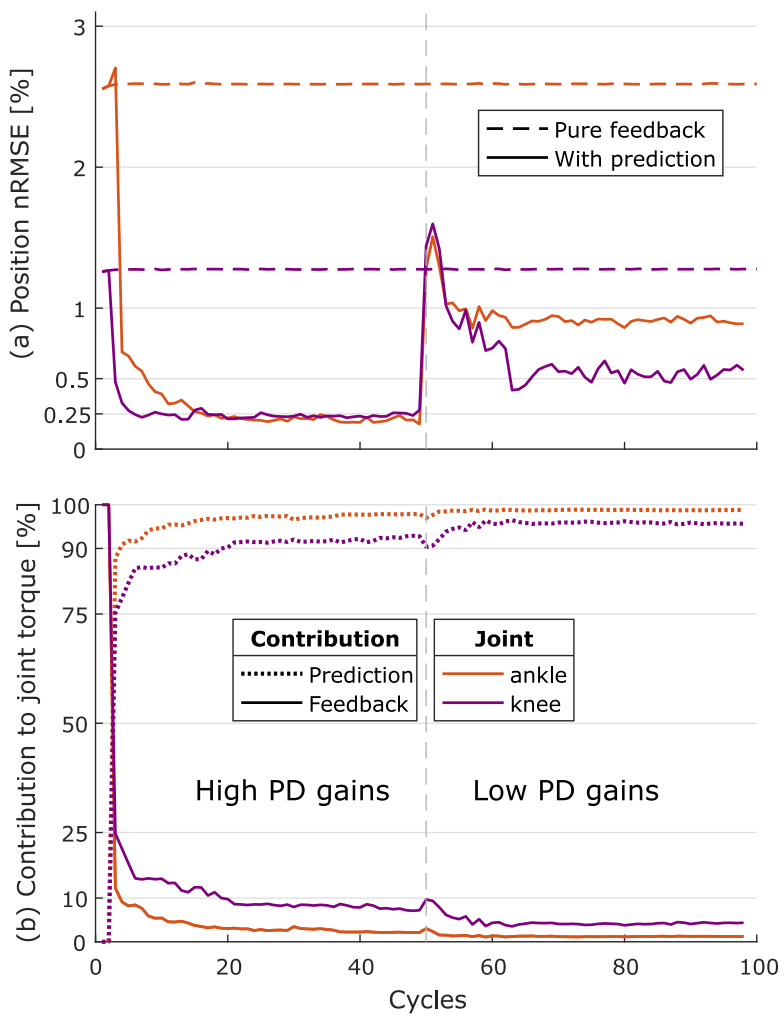

Fig. 3. Experiment 2.A: (a) Evolution of the nRMSE of the knee and ankle joint positions per cycle. The nRMSEs are compared to the ones obtained in pure feedback mode with high gains (dashed). (b) Corresponding contributions of the predictive torque and feedback torque components to the total joint torques. The PD feedback gains were lowered after 50 gait cycles of learning.

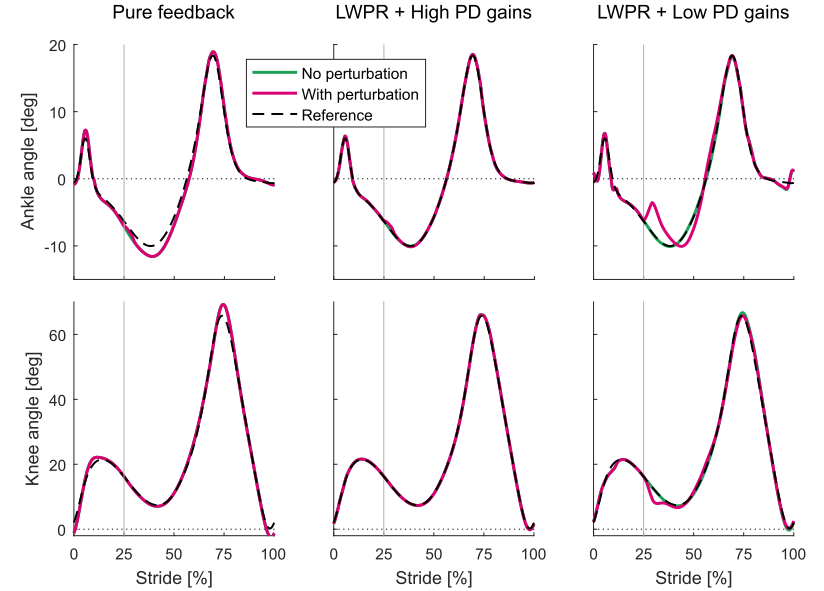

Fig. 4. Experiment 2.B: Position profile of the ankle (top row) and knee (bottom row). The left column shows the pure feedback mode with high gains, and the central (resp. right) column shows the LWPR + high (resp. low) PD feedback gains mode, with $K_{\mathrm{FB}}^{i}=100 \%$ (resp. $K_{\mathrm{FB}}^{i}=10 \%$ ). All panels compare the unperturbed behavior (green) and the behavior induced after a torque perturbation applied at $\phi_{\text {pert }}=25 \%$ (purple).

reports the evolution of the contributions of the predictive and feedback torque components to the total joint torque per cycle. It shows that the feedback contribution to the joint torque reaches $2.15 \%$ for the ankle joint and $7.40 \%$ for the knee joint before lowering the PD gains; these values then decrease to $1.22 \%$ for the ankle joint and to $4.28 \%$ for the knee joint with reduced PD gains. During the simulation, 50 and 78 RFs were created by the LWPR-based module for the ankle model and the knee model respectively.

Next, we conducted Experiment 2.B, a perturbation study assessing the prosthesis resulting impedance. Figure 4 shows the ankle and knee angle profiles in three cases: in the pure feedback mode, and when the predictive controller is supplemented with high and low feedback gains. It further compares the unperturbed profile (steady-state) to the profile obtained when a torque perturbation is applied at $\phi_{\text {pert }}=25 \%$, i.e. during the stance phase. This figure confirms that the predictive action was beneficial for accurate trajectory tracking, both with high and low feedback gains. It further illustrates that both joints had lower impedance (i.e., were more compliant) in the "low PD gains" condition than in both others. We obtained similar results for perturbations applied at three other phases of the gait cycle, see Table II. In sum, lowering the PD gains enhanced the simulated prosthesis compliance, with limited impact regarding position tracking.

TABLE II

JOINT MAX. DISPLACEMENT AFTER TORQUE PERTURBATIONS (DEGREE)

\begin{tabular}{c||c||c|c|c|c|}
\cline { 2 - 6 } \multicolumn{1}{c|}{} & $\phi_{\text {pert }}[\%$ stride] & 0 & 25 & 50 & 75 \\
\hline \hline \multirow{4}{*}{ Ankle } & pure FB & 1.02 & 0.52 & 0.61 & 1.08 \\
& high-gains & 0.97 & 0.62 & 0.62 & 1.01 \\
& low-gains & 6.81 & 4.63 & 4.44 & 6.98 \\
\hline \multirow{3}{*}{ Knee } & pure FB & 0.19 & 0.08 & 0.09 & 0.14 \\
& high-gains & 0.30 & 0.22 & 0.22 & 0.38 \\
& low-gains & 7.20 & 3.85 & 1.96 & 4.43 \\
\hline
\end{tabular}




\section{Experiment 3: Cadence switching}

Results of the cadence switching experiment showed that the proposed control architecture allows to maintain a stable behavior when switching from one gait cadence to another. The controller architecture is indeed able to make fast adjustments following a change in walking cadence, as emphasized in Fig. 5. After the initial learning phase of the dynamical internal models of the joints, the torque computed by the LWPR-based module is accurately predicted for the three gait cadences and for both joints. This is illustrated in Fig. 6, depicting the ankle and knee torque profiles averaged over the last 5 cycles of the last simulation stage associated to each gait cadence. The mean nRMSE between the torque predicted by the ankle internal model and the total ankle torque over the last 5 cycles of each gait cadence is $0.76 \%, 0.59 \%$ and $0.89 \%$ for slow, normal and fast walking respectively. Similarly, the corresponding nRMSE values for the knee predictive torque are respectively $0.70 \%$, $0.98 \%$ and $1.72 \%$. This accurate prediction allows for precise position tracking performance for both joints, as illustrated by the averaged joint angle profiles shown in the same figure for the three gait cadences. Indeed, the nRMSE between the joints positions and their reference trajectories remained below the value observed with a pure feedback control approach
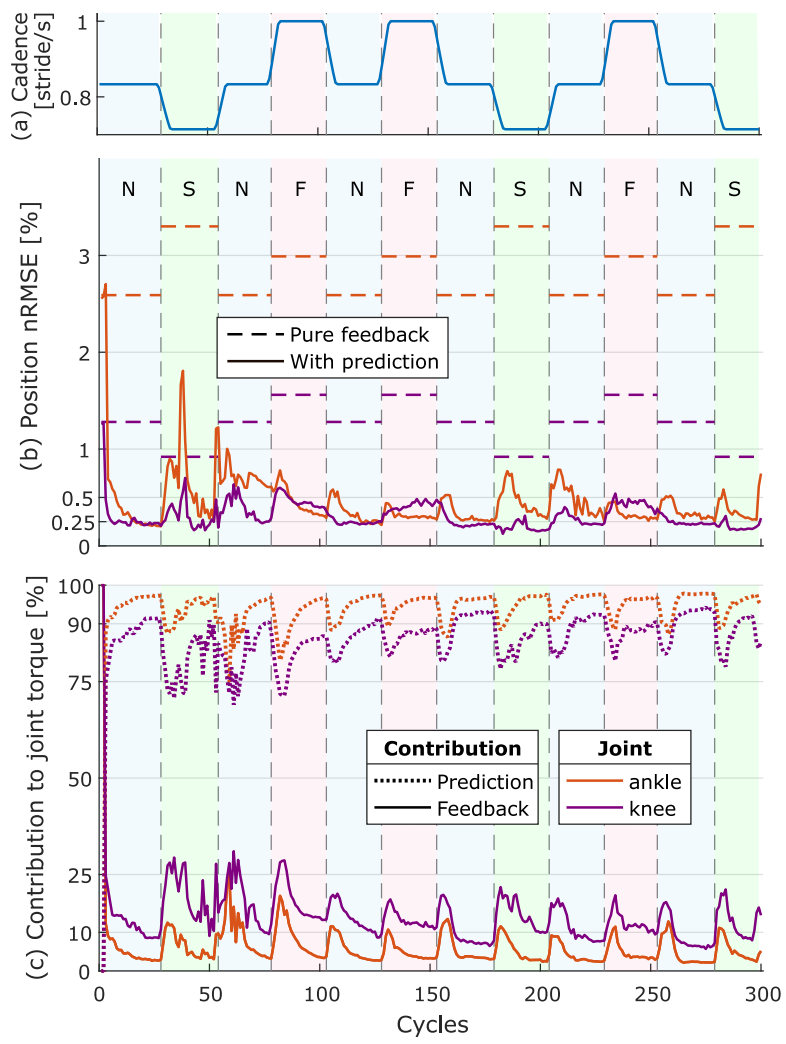

Fig. 5. Experiment 3: adaptation to changing cadence. (a) Target cadence with smooth transitions corresponding to $\mathrm{S}=$ Slow; $\mathrm{N}=$ Normal; and $\mathrm{F}=$ Fast. (b) Evolution of the nRMSE of the knee and ankle joint positions per cycle and (c) corresponding contributions of the predictive torque $\tau_{\text {pred }}^{i}$ and feedback torque $\tau_{\mathrm{FB}}^{i}$ components to the total joint torques. The gait cadence was changed every 25 gait cycles with smooth transitions over the first 5 cycles. The nRMSEs depicted in (b) are compared to the ones obtained with a pure feedback controller ( $\tau_{\text {pred }}^{i}=0$, dashed) in steady-sate. (i.e. with $\tau_{\text {pred }}^{i}=0$ for both joints and for the three walking speeds). In the last simulation stage associated with each gait cadence, the mean nRMSE of the ankle angle over the last 5 cycles reached $0.28 \%, 0.29 \%$ and $0.28 \%$ for slow, normal and fast walking respectively. This was associated to a respective feedback contribution to the total ankle torque of $2.88 \%$, $2.20 \%$ and $3.28 \%$. Similar results were obtained for the knee joint: in the last simulation stage associated with each gait cadence, the mean nRMSE of the knee angle over the last 5 cycles was $0.18 \%, 0.23 \%$ and $0.39 \%$ for slow, normal and fast walking respectively. This was associated to a mean feedback contribution to the total knee torque of $10.08 \%, 6.24 \%$ and $10.36 \%$ respectively. By the end of the simulation, 266 and 418 RFs had been created by the LWPR-based module for the ankle and the knee models respectively.

These results show that the control architecture is robust to changing gait cadences and that the LWPR-based module is able to continuously learn the dynamical internal models of the knee and ankle joints.

\section{DISCUSSION AND CONCLUSION}

We developed an adaptive control architecture for a transfemoral prosthesis combining a predictive torque command with a feedback error-correction mechanism. The predictive action uses the LWPR algorithm, acting as a dynamical internal model of the prosthesis system. We evaluated the proposed approach on a simple biped model in a simulation environment. Results showed that the LWPR module is able to quickly learn the dynamical model of each joint and provides accurate torque commands in real-time. This allows decreasing the feedback gains, leading to similar position tracking performance, but with lower joint impedance. Interestingly, the set of inputs of the LWPR module only includes joint angular position and velocity signals, i.e., signals that are readily available from typical encoders of a real device. This suggests that we may not need to feed the LWPR models with a measurement of the ground contact forces to obtain a reasonable approximation of the dynamical internal models. Although this result has yet to be validated in a real-world experiment, it is consistent with [36], where the dynamical model of a robot arm manipulating unknown objects was accurately learned based on the joint trajectories and achieved precise control during load manipulation tasks, even with nonstationary loads.

The proposed control architecture is bio-inspired, i.e., (i) it implements artificial Central Pattern Generators (CPGs) using an adaptive oscillator [37], [38], and (ii) it partially mimics the function of the cerebellum in human locomotion. Indeed, previous studies on human motor control highlighted the key role of the cerebellum in motor control and learning, and support the assumption that this neural structure encodes forward and inverse internal models [39] to achieve accurate and coordinated movements [40]. In this way, the putative role of the cerebellum is twofold: (1) it provides a feedforward predictive action based on continuous learning of the task, and (2) it provides an error-correction action to ensure robustness to perturbations [41]. Our controller includes a 

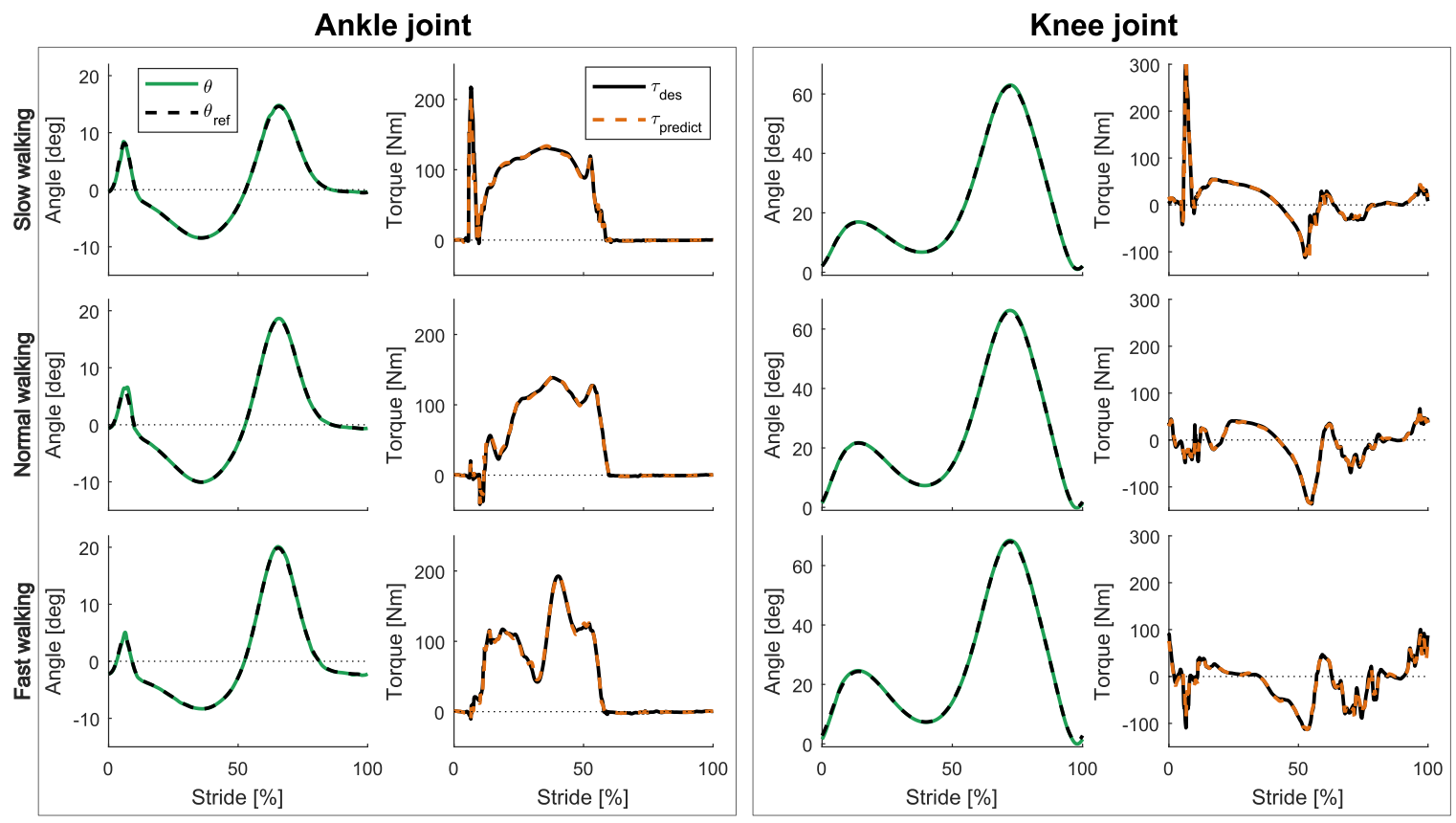

Fig. 6. Experiment 3: Ankle angles and torques over the gait cycle, averaged over the last 5 cycles of the last simulation stage associated to each gait cadence. Left to right: Ankle angle and torque profiles, and knee angle and torque profiles. Top to bottom: slow, normal and fast walking cadences.

prediction module encoding the dynamical internal models of the prosthesis joints. As future work, adaptive short-term cerebellar corrective actions will be added in the controller (similarly as in [26], [27], [42]) to fully exploit adaptation skills based on feedback from actual movement.

In addition, we showed that this controller is robust to changing gait cadences and that the LWPR-based module can continuously learn the dynamical internal model of the knee and ankle joints. This suggests that the LWPR module has the potential to automatically and continuously adapt these models to each user and locomotion activity. Other contributions targeted the development of online adaptive controllers for locomotion. The approach presented in [18] used the metabolic cost of walking - which is characterized by oxygen consumption - to adapt the assistive torque profile provided by an ankle exoskeleton. Another human-in-the-loop optimization method [17] used muscle activity signals to adapt the torque pattern delivered by an ankle exoskeleton. Both studies illustrated that it is possible to optimize the humanrobot synergy by adapting the device control parameters as a function of metabolic signals collected in real-time. We also targeted closed-loop adaptation of a locomotion assistive device, not for optimizing the human metabolic cost, but rather for enhancing the compliance of the device. Interestingly, our approach required only the use of proprioceptive sensors embedded in the device itself. In future work, we will investigate if there exist a relationship between this intrinsic compliance of the device, and thus the way it reacts to natural stride-tostride variability, and the resulting metabolic cost of the user.

The presented study has also some limitations. First, the simulated biped walker has no forward velocity and acceleration, since its center of mass is constrained to move only in the vertical direction and the implemented ground contact model only includes the vertical component of the GRF. This causes significant differences compared to reality, e.g. in the dynamic force profiles at the feet. Additionally, the transfemoral prosthesis was modelled as having the same dimensions, mass and inertia than the sound leg and with an ideal controller, assuming perfect torque tracking. These simplifications are deemed reasonable given the objectives of the simulation experiments, but they should be kept in mind when translating the presented work into real-life applications.

The experiment showing the robustness to changing cadence was tested with only three steady-state conditions (and smooth transitions in-between). Further experiments are required to validate the algorithm performance over a more continuous set of target cadences. Moreover, the model complexity to be learned by the LWPR significantly increased in this third experiment, as illustrated by the higher number of RFs generated at the end of this simulation. In future work, we will investigate the design of a mechanism to couple both estimation and tracking errors toward more efficient learning and RFs generation process [43]. As an alternative to LWPR, we will explore other algorithms (i.e., LGR [44]) in which the local models do know each other and collaborate to generate a function fit, such that going back to a previously learned region of the state-space would draw exclusively from the past learning.

To sum up, an online adaptive learning of the dynamical model of a biarticular prosthesis allows to provide accurate torque commands without the need for an analytical model of the human+prosthesis complex, whose derivation would be a complicated task. The simulation experiments presented in this paper indicate that the approach holds the potential to provide adaptive and robust control for lower-limb prostheses. Future work will focus on assessing the performance of the itera- 
tive learning mechanism on a real prosthesis, by conducting walking experiments with amputated subjects.

\section{REFERENCES}

[1] S. R. Wurdeman, P. M. Stevens, and J. H. Campbell, "Mobility Analysis of AmpuTees (MAAT I): Quality of life and satisfaction are strongly related to mobility for patients with a lower limb prosthesis," Prosthetics and Orthotics International, vol. 42(5), pp. 498-503, 2018.

[2] P. Jefferies, P. Gallagher, and M. Philbin, "Being "just normal": a grounded theory of prosthesis use," Disability and rehabilitation, vol. 40, pp. 1-10, Apr. 2017.

[3] E. C. Baars, E. Schrier, P. U. Dijkstra, and J. H. Geertzen, "Prosthesis satisfaction in lower limb amputees: A systematic review of associated factors and questionnaires," Medicine, vol. 97(39), 2018.

[4] Ottobock, "C-Leg 4 above knee prosthetic leg," URL: https://www.ottobockus.com/prosthetics/lower-limbprosthetics/solution-overview/c-leg-above-knee-system/, Visited on Jan. 17, 2020

[5] Össur, "Proprio Foot," URL: https://www.ossur.com/enus/prosthetics/feet/proprio-foot, Visited on Feb. 28, 2020.

[6] R. L. Waters, J. Perry, D. Antonelli, and H. Hislop, "Energy cost of walking of amputees: the influence of level of amputation," The Journal of Bone and Joint Surgery. American Volume, vol. 58(1), pp. 42-46, 1976.

[7] R. L. Waters and S. Mulroy, "The energy expenditure of normal and pathologic gait," Gait \& Posture, vol. 9(3), pp. 207-231, 1999.

[8] W. C. Miller, A. B. Deathe, M. Speechley, and J. Koval, "The influence of falling, fear of falling, and balance confidence on prosthetic mobility and social activity among individuals with a lower extremity amputation," Archives of Physical Medicine and Rehabilitation, vol. 82(9), pp. 1238-1244, 2001.

[9] A. M. Grabowski and S. D'Andrea, "Effects of a powered ankle-foot prosthesis on kinetic loading of the unaffected leg during level-ground walking," Journal of NeuroEngineering and Rehabilitation, vol. 10, p. 49, 2013.

[10] H. M. Herr and A. M. Grabowski, "Bionic ankle-foot prosthesis normalizes walking gait for persons with leg amputation," Proceedings. Biological Sciences, vol. 279(1728), pp. 457-464, 2012.

[11] M. R. Tucker, J. Olivier, A. Pagel, H. Bleuler, M. Bouri, O. Lambercy, J. d. R. Millán, R. Riener, H. Vallery, and R. Gassert, "Control strategies for active lower extremity prosthetics and orthotics: a review," Journal of NeuroEngineering and Rehabilitation, vol. 12, 2015.

[12] F. Sup, A. Bohara, and M. Goldfarb, "Design and Control of a Powered Transfemoral Prosthesis:," The International Journal of Robotics Research, 2008.

[13] V. Azimi, T. T. Nguyen, M. Sharifi, S. A. Fakoorian, and D. Simon, "Robust Ground Reaction Force Estimation and Control of Lower-Limb Prostheses: Theory and Simulation," IEEE Transactions on Systems, Man, and Cybernetics: Systems, pp. 1-12, 2018.

[14] D. Quintero, D. J. Villarreal, D. J. Lambert, S. Kapp, and R. D. Gregg, "Continuous-Phase Control of a Powered Knee-Ankle Prosthesis: Amputee Experiments Across Speeds and Inclines," IEEE Transactions on Robotics, vol. 34(3), pp. 686-701, 2018.

[15] S. Huang, J. P. Wensman, and D. P. Ferris, "An Experimental Powered Lower Limb Prosthesis Using Proportional Myoelectric Control,' Journal of Medical Devices, vol. 8(2), 2014.

[16] S. Heins, L. Flynn, J. Geeroms, D. Lefeber, and R. Ronsse, "Torque control of an active elastic transfemoral prosthesis via quasi-static modelling," Robotics and Autonomous Systems, vol. 107, pp. 100-115, 2018.

[17] R. W. Jackson and S. H. Collins, "Heuristic-Based Ankle Exoskeleton Control for Co-Adaptive Assistance of Human Locomotion," IEEE Transactions on Neural Systems and Rehabilitation Engineering, vol. 27(10), pp. 2059-2069, 2019.

[18] J. Zhang, P. Fiers, K. A. Witte, R. W. Jackson, K. L. Poggensee, C. G. Atkeson, and S. H. Collins, "Human-in-the-loop optimization of exoskeleton assistance during walking," Science, vol. 356(6344), pp. 1280-1284, 2017.

[19] N. Hogan, "Adaptive control of mechanical impedance by coactivation of antagonist muscles," IEEE Transactions on Automatic Control, vol. 29(8), pp. 681-690, 1984.

[20] M. L. Latash and V. M. Zatsiorsky, "Joint stiffness: Myth or reality?" Human Movement Science, vol. 12(6), pp. 653-692, 1993.
[21] A. D. Kuo, "The Relative Roles of Feedforward and Feedback in the Control of Rhythmic Movements," Motor Control, vol. 6(2), pp. 129$145,2002$.

[22] D. Nguyen-Tuong and J. Peters, "Learning Robot Dynamics for Computed Torque Control Using Local Gaussian Processes Regression,' in 2008 ECSIS Symposium on Learning and Adaptive Behaviors for Robotic Systems (LAB-RS), 2008, pp. 59-64.

[23] S. Schaal and N. Schweighofer, "Computational motor control in humans and robots," Current Opinion in Neurobiology, vol. 15(6), pp. 675-682, 2005

[24] J. Sun de la Cruz, D. Kulic, and W. Owen, "Learning inverse dynamics for redundant manipulator control," in 2010 International Conference on Autonomous and Intelligent Systems, AIS 2010, 2010, pp. 1-6.

[25] S. Heins and R. Ronsse, "Compliant control of a transfemoral prosthesis combining predictive learning and primitive-based reference trajectories," in The 5th International Symposium on Wearable Robotics (WeRob 2020), 2020, in press.

[26] S. Tolu, M. Vanegas, N. R. Luque, J. A. Garrido, and E. Ros, "Bioinspired adaptive feedback error learning architecture for motor control," Biological Cybernetics, vol. 106(8), pp. 507-522, 2012.

[27] E. Massi, L. Vannucci, U. Albanese, M. C. Capolei, A. Vandesompele, G. Urbain, A. M. Sabatini, J. Dambre, C. Laschi, S. Tolu, and E. Falotico, "Combining Evolutionary and Adaptive Control Strategies for Quadruped Robotic Locomotion," Frontiers in Neurorobotics, vol. 13, p. 71, 2019.

[28] D. A. Winter, The biomechanics and motor control of human gait. University of Waterloo Press, 1987.

[29] T. Yan, A. Parri, V. Ruiz Garate, M. Cempini, R. Ronsse, and N. Vitiello, "An oscillator-based smooth real-time estimate of gait phase for wearable robotics," Autonomous Robots, vol. 41(3), pp. 759-774, 2017.

[30] H. Laloyaux and R. Ronsse, "Extraction of simple monophasic motor primitives towards bio-inspired locomotion assistance," in 2019 IEEE International Conference on Cyborg and Bionic Systems (CBS), 2019, pp. 253-260.

[31] S. Vijayakumar, A. D'Souza, and S. Schaal, "Incremental online learning in high dimensions," Neural Computation, vol. 17(12), pp. 2602-2634, 2005.

[32] N. Docquier, A. Poncelet, and P. Fisette, "ROBOTRAN: a powerful symbolic gnerator of multibody models," Mechanical Sciences, vol. 4 pp. 199-219, 2013.

[33] “Robotran - Home," URL: https://www.robotran.be/, Visited on Oct. 1, 2020.

[34] H. Geyer and H. Herr, "A Muscle-Reflex Model That Encodes Principles of Legged Mechanics Produces Human Walking Dynamics and Muscle Activities," IEEE Transactions on Neural Systems and Rehabilitation Engineering, vol. 18(3), pp. 263-273, 2010.

[35] S. Klanke, S. Vijayakumar, and S. Schaal, "A Library for Locally Weighted Projection Regression," Journal of Machine Learning Research, vol. 9, pp. 623-626, 2008.

[36] G. Petkos and S. Vijayakumar, "Load estimation and control using learned dynamics models," in 2007 IEEE/RSJ International Conference on Intelligent Robots and Systems, 2007, pp. 1527-1532.

[37] L. Righetti, J. Buchli, and A. J. Ijspeert, "Dynamic Hebbian learning in adaptive frequency oscillators," Physica D: Nonlinear Phenomena, vol. 216(2), pp. 269-281, 2006.

[38] R. Ronsse, T. Lenzi, N. Vitiello, B. Koopman, E. van Asseldonk, S. M. M. De Rossi, J. van den Kieboom, H. van der Kooij, M. C. Carrozza, and A. J. Ijspeert, "Oscillator-based assistance of cyclical movements: model-based and model-free approaches," Medical \& Biological Engineering \& Computing, vol. 49(10), pp. 1173-1185, 2011.

[39] S. Tolu, M. C. Capolei, L. Vannucci, C. Laschi, E. Falotico, and M. V. Hernández, "A Cerebellum-Inspired Learning Approach for Adaptive and Anticipatory Control," International Journal of Neural Systems, vol. 30(1):1950028, 2020.

[40] M. Kawato, "Internal models for motor control and trajectory planning," Current Opinion in Neurobiology, vol. 9(6), pp. 718-727, 1999.

[41] I. Pisotta and M. Molinari, "Cerebellar contribution to feedforward control of locomotion," Frontiers in Human Neuroscience, vol. 8, 2014

[42] M. C. Capolei, E. Angelidis, E. Falotico, H. H. Lund, and S. Tolu, "A Biomimetic Control Method Increases the Adaptability of a Humanoid Robot Acting in a Dynamic Environment," Frontiers in Neurorobotics, vol. 13, 2019.

[43] J. Nakanishi, J. A. Farrell, and S. Schaal, "Composite adaptive control with locally weighted statistical learning," Neural Networks, vol. 18, no. 1, pp. 71-90, 2005

[44] J.-A. Ting, F. Meier, S. Vijayakumar, and S. Schaal, Locally Weighted Regression for Control. Boston, MA: Springer US, 2016, pp. 1-14. 